\title{
Effect of Radiation on the Unsteady Hydro magnetic Heat Transfer Flow in a Vertical Wavy Channel with Oscillating Flux
}

\author{
P. Raveendra Nath ${ }^{1,}$ N. B. V. Rama deva prasad ${ }^{2}$, B. Sreenivasa Reddy ${ }^{3}$ \\ P. M. V. Prasad ${ }^{4}$ \\ ${ }^{1}$ Lecturer in Mathematics,Department of Mathematics, Sri Krishnadevaraya University College of \\ Engineering and Technology, S. K. University, Anantapur - 515 055, A.P., India. \\ ${ }^{2}$ Lecturer in Mathematics, Balaji P.G.College, Anantapur,Andhra Pradesh,India. \\ ${ }^{3}$ Assistant Professor, Department of Mathematics, Yogi vemana University, Kadapa, Andhra Pradesh,India. \\ ${ }^{4}$ Department of Mathematics, S.V.G.S. Degree College, Nellore, A.P., India.
}

\begin{abstract}
In this paper we study the unseady Convective Heat Transfer flow of a viscous electrically conducting fluid in a vertical wavy Channel under the influence of an inclined magnetic field. The unsteadiness in the flow is due to an Oscillatory flux in the fluid region. The equations governing the flow and Heat Transfer which are Non-linear coupled in nature are solved by employing a perturbation technique with the slope $\delta$ of the wavy walls as perturbation parameter the influence of Hall effects the radiation and Heat sources on the flow and Heat Transfer characteristics has been studied graphically the average Nusselt Number on the boundary walls $\eta= \pm 1$ are numerically evaluated for different values of, $\beta, \lambda$ and $N$.
\end{abstract}

\section{Introduction}

Flows which arise due to the interaction of the gravitational force and density differences caused by the simultaneous diffusion of thermal energy have many applications in geophysics and engineering. Such thermal and mass diffusion plays a dominant role in a number of technological and engineering systems.

Obviously, the understanding of this transport process is desirable in order to effectively control the overall transport characteristics. The combined effect of thermal and mass diffusion in channel flows has been studied by a few authors in recent times [1]. The problem of combined buoyancy driven thermal and mass diffusion has been studied in parallel plate geometries by a few authors, notably, Lai [2], Chen and Mont Sogol [3], Poulikakos [4] and Angirasa et. al., [5].

Unsteady convection flows play an important role in aerospace technology, turbomachinery and chemical engineering. Such flows arise due to either unsteady motion of a boundary temperature. Unsteadiness may also be due to oscillatory free stream velocity or temperature. These oscillatory free convective flows are important from technological point of view.

Vajravelu have investigated the influence of the wall waviness on friction and pressure drop of the wall waviness on friction and pressure drop of the generalized couette flow. The MHD effects on the oscillating convection flows have been discussed by several authors [6].Ching-Yang Chang [7] has analyzed the convective heat and mass transfer near a vertical wavy surface embedded in a fluid saturated porous medium.

The interaction of radiation with laminar free convection heat transfer from a vertical plate was investigated by Cess [8] for an absorbing, emitting fluid in the optically thick region, using the singular perturbation technique. Hossain and Takhar [6] studied the radiation effects on mixed convection along a vertical plate with uniform surface temperature using the Keller Box finite difference method. In all these papers the flow is considered to be steady.

\section{Formulation of the Problem}

We consider the unsteady flow of an incompressible, viscous ,electrically conducting fluid through a porous medium confined in a vertical channel bounded by two wavy walls under the influence of an inclined magnetic field of intensity Ho lying in the plane (y-z).The unsteadiness is due to an oscillatory flux The magnetic field is inclined at an angle $\alpha_{1}$ to the axial direction and hence its components are $\left(0, H_{0} \operatorname{Sin}\left(\alpha_{1}\right), H_{0} \operatorname{Cos}\left(\alpha_{1}\right)\right)$.In view of the waviness of the wall the velocity field has components $(\mathrm{u}, 0, \mathrm{w})$ The magnetic field in the presence of fluid flow induces the current $\left(J_{x}, 0, J_{z}\right)$. We choose a rectangular cartesian co-ordinate system $\mathrm{O}(\mathrm{x}, \mathrm{y}, \mathrm{z})$ with $\mathrm{z}$-axis in the vertical direction and the walls at ${ }_{x= \pm f\left(\frac{\delta z}{L}\right)}$.

The equations governing the flow and Heat transfer taking Hall Currents into consideration are 


$$
\begin{aligned}
& \frac{\partial u}{\partial t}+u \frac{\partial u}{\partial x}+w \frac{\partial u}{\partial z}=-\left(\frac{\partial p}{\partial x}\right)+\mu\left(\frac{\partial^{2} u}{\partial x^{2}}+\frac{\partial^{2} u}{\partial z^{2}}\right) \\
& -\left(\frac{\sigma \mu_{e} H_{00}^{2} \operatorname{Sin}^{2}(\alpha)}{1+m^{2} H_{0}^{2} \operatorname{Sin}^{2}(\alpha)}\right)\left(u+m H_{0} w \operatorname{Sin}(\alpha)\right) \\
& \frac{\partial w}{\partial t}+u \frac{\partial w}{\partial x}+w \frac{\partial w}{\partial z}=-\left(\frac{\partial p}{\partial z}\right)+\mu\left(\frac{\partial^{2} w}{\partial x^{2}}+\frac{\partial^{2} w}{\partial z^{2}}\right) \\
& -\left(\frac{\sigma \mu_{e} H_{0}^{2} \operatorname{Sin}^{2}(\alpha)}{1+m^{2} H_{0}^{2} \operatorname{Sin}^{2}(\alpha)}\right)\left(w-m H_{0} u \operatorname{Sin}(\alpha)\right)+\beta g\left(T-T_{e}\right) \\
& \rho_{o} C_{p}\left(\frac{\partial T}{\partial t}+u \frac{\partial T}{\partial x}+w \frac{\partial T}{\partial z}\right)=k_{f}\left(\frac{\partial^{2} T}{\partial x^{2}}+\frac{\partial^{2} T}{\partial z^{2}}\right)+Q-\left(\frac{\partial\left(q_{r}\right)}{\partial x}\right)
\end{aligned}
$$

The flow is maintained by a constant volume flux for which a characteristic velocity is defined as

$$
q\left(1+e^{i \omega t}\right)=\frac{1}{L} \int_{-L f}^{L f} w d x
$$

The boundary conditions are

$\mathrm{u}=0, \mathrm{w}=0 \quad \mathrm{~T}=\mathrm{T}_{1} \quad$ on $\quad x=-f\left(\frac{\delta z}{L}\right)$

$\mathrm{u}=0, \quad \mathrm{w}=0, \quad \mathrm{~T}=\mathrm{T}_{2} \quad$ on $\quad x=f\left(\frac{\delta z}{L}\right)$

Eliminating the pressure from equations(2.1)\&(2.2) and introducing the Stokes Stream function $\psi$ as

$$
u=-\left(\frac{\partial \psi}{\partial z}\right), w=\left(\frac{\partial \psi}{\partial x}\right)
$$

and using Rosseland approximation $\psi$ is

$$
\begin{aligned}
& \frac{\partial\left(\nabla^{2} \psi\right)}{\partial t}+\left(\frac{\partial \psi}{\partial z}\right)\left(\frac{\partial\left(\nabla^{2} \psi\right)}{\partial x}\right)-\left(\frac{\partial \psi}{\partial x}\right)\left(\frac{\partial\left(\nabla^{2} \psi\right)}{\partial z}\right)=\mu \nabla^{4} \psi+\beta g \frac{\partial\left(T-T_{e}\right)}{\partial x}- \\
& -\left(\frac{\sigma \mu_{e}^{2} H_{0}^{2} \operatorname{Sin}^{2}(\alpha)}{1+m^{2} H_{0}^{2} \operatorname{Sin}^{2}(\alpha)}\right) \nabla^{2} \psi \\
& \rho_{o} C_{p}\left(\frac{\partial \psi}{\partial x} \frac{\partial T}{\partial z}-\frac{\partial \psi}{\partial z} \frac{\partial T}{\partial x}\right)=K_{f} \nabla^{2} \theta+Q+\left(\frac{16 \sigma^{*} T_{e}^{3}}{3 \beta_{R}}\right)\left(\frac{\partial^{2} T}{\partial x^{2}}\right)
\end{aligned}
$$

On introducing the following Non-dimensional variables

$$
\left(x^{\prime}, z^{\prime}\right)=\frac{(x, z)}{L}, \psi^{\prime}=\frac{\psi}{q L}, \theta=\frac{T-T_{2}}{T_{1}-T_{2}}
$$

the equation of momentum and energy in the non-dimensional form are

$$
\begin{aligned}
& \nabla^{4} \psi-M_{1}^{2} \nabla^{2} \psi+\frac{G}{R}\left(\frac{\partial \theta}{\partial x}\right)=\gamma^{2} \frac{\partial\left(\nabla^{2} \psi\right)}{\partial t}+R\left(\frac{\partial \psi}{\partial z} \frac{\partial\left(\nabla^{2} \psi\right)}{\partial x}-\frac{\partial \psi}{\partial x} \frac{\partial\left(\nabla^{2} \psi\right)}{\partial z}\right) \\
& P \gamma^{2} \frac{\partial T}{\partial t}+R\left(\frac{\partial \psi}{\partial x} \frac{\partial \theta}{\partial z}-\frac{\partial \psi}{\partial z} \frac{\partial \theta}{\partial x}\right)=\nabla^{2} \theta+\alpha+\frac{4}{3 N_{1}} \frac{\partial^{2} \theta}{\partial x^{2}}
\end{aligned}
$$

Where

$$
\begin{array}{cl}
G=\frac{\beta g \Delta T_{e} L^{3}}{v^{2}} & \text { (Grashof Number) } \\
M^{2}=\frac{\sigma \mu_{e}^{2} H_{o}^{2} L^{2}}{v^{2}} & \text { (Hartman Number) } \\
M_{1}^{2}=\frac{M^{2} \operatorname{Sin}^{2} \alpha}{1+m^{2} \operatorname{Sin}^{2} \alpha} & \\
R=\frac{q L}{v} & \text { (Reynolds Number) }
\end{array}
$$




$$
\begin{array}{cl}
P=\frac{\mu C_{p}}{K_{f}} & \text { (Prandtl Number) } \\
\alpha=\frac{Q L^{2}}{K_{f}\left(T_{1}-T_{2}\right)} & \text { (Heat source parameter) } \\
N_{1}=\frac{\beta_{R} K_{f}}{4 \sigma^{*} T_{e}^{3}} & \text { (Radiation parameter) }
\end{array}
$$

The corresponding boundary conditions are

$$
\begin{aligned}
& \psi(+1)-\psi(-1)=1 \\
& \frac{\partial \psi}{\partial z}=0, \frac{\partial \psi}{\partial x}=0, \theta=1, \quad \text { at } x=-f(\delta z) \\
& \frac{\partial \psi}{\partial z}=0, \frac{\partial \psi}{\partial x}=0, \theta=0, \quad \text { at } x=+f(\delta z)
\end{aligned}
$$

\section{Nusselt Number}

The rate of heat transfer (Nusselt Number) on the boundaries is given by

$$
q=-k\left(\frac{\partial T}{\partial x}\right)_{x= \pm L f}
$$

and the corresponding expressions in the non-dimensional form are

$$
\begin{aligned}
& (N u)_{\eta=+1}=\left(\frac{d_{3}+\delta d_{5}}{f\left(\theta_{m}-1\right)}\right) \\
& (N u)_{\eta=-1}=\left(\frac{d_{4}+\delta d_{6}}{f\left(\theta_{m}-h_{1}\right)}\right)
\end{aligned}
$$

where

$$
\theta_{m}=\frac{1}{L} \int_{-L f}^{L f} \theta d \eta
$$

\section{Results and Discussion}

In this analysis we investigate the combined influence of Hall Currents and Radiation effect on unsteady convective heat transfer flow of a viscous electrically conducting fluid in a vertical wavy channel with oscillatory flux. The analysis has been carried out by perturbation method with slope $\delta$ as a perturbation parameter with $\mathrm{P}=0.71$ and $\delta=0.01$. The secondary velocity u, axial velocity $\mathrm{w}$, Non-dimensional temperature $\theta$ is shown in Figs.1-9. For different values of $\beta, \lambda$, and $N$.

The secondary velocity which is due to the waviness of the boundary is exhibited in Figs.1-3.The influence of waviness of boundaries of $\mathrm{u}$ is shown in Fig.1, higher the constriction of the channel walls smaller $|\mathrm{u}|$ in the flow region (Fig.1). The effect on inclination ' $\lambda$ ' of $\mathrm{u}$ is shown in Fig.2. It is observed that higher the inclination of the magnetic field smaller $|u|$ in the flow region (Fig.2). Fig. 3 shows that the variation of $u$ with Radiation parameter $\mathrm{N}$ shows that an increase in $\mathrm{N}$ results in a marginally increment in $|\mathrm{u}|$.

Fig. 4 shows that the variation of $w$ with constructive parameter $\beta$. It is found that the reversal flow which appears $|\beta|=0.3$ disappear with higher values of $|\beta|$ higher the constriction of the channel walls larger $|\mathrm{w}|$ in the flow region (Fig.4). The variation of ' $\mathrm{w}$ ' with inclination of magnetic field $\lambda$ is shown in Fig.5 It is found that the reversal flow which occurs at $\lambda=0.5$ in the flow region except at $\eta= \pm 0.8$ disappear for higher inclinations. $|\mathrm{w}|$ reduces with increase in $\lambda \leq 0.5$ and enhances with higher $\lambda=0.75$ and again reduces with further increase in the inclination of magnetic field (Fig.5). From Fig.6. It is found that the reversal flow appears in the

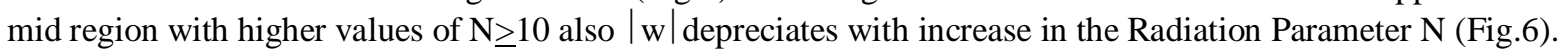
The influence of the surface geometry in $\theta$ is exhibited in Fig.7. It is found that higher the constriction of the channel walls larger the actual temperature in the flow region. The effect of inclination of the magnetic field on $\theta$. It is found that an increase in the inclination of the magnetic field results in an enhancement with increase in the actual temperature for $\lambda=0.5, \theta<0$ in the left half and $\theta>0$ in right half (Fig.8). 
The effect of radiative Heat transfer on $\theta$ is shown in Fig.9 for $\theta= \pm 1.5, \theta<0$ in left half end positive in the right half while for higher $\mathrm{N}>2.5, \theta>0$ is positive in the entire region. An increase in the radiation parameter results and an enhancement in the actual temperature of its flow region.

The average Nusselt Number $(\mathrm{Nu})$ which measures the rate of Heat transfer at the boundaries $\eta= \pm 1$ is shown in Tables 1 and 2 .For different values of $\beta$ and $\lambda$. The variation of $\mathrm{Nu}$ with $\beta$ shows that higher the constriction of the channel wavy $(|\beta| \leq 0.5)$, larger $|\mathrm{Nu}|$ at $\eta=+1$ and for further increases in $(|\beta| \leq 0.7)$. We notice a depreciation $|\mathrm{Nu}|$ at $\eta=+1$ while at $\eta=-1$ the rate of Heat transfer enhances with increase in $|\beta|$ Also an increase in the inclination of the magnetic field leads to an enhancement in $|\mathrm{Nu}|$ at both the wavy. In general we notice that the rate of Heat transfer at the right boundary is much higher than that at the left boundary $\eta=-$ 1(Tables.1 and 2).

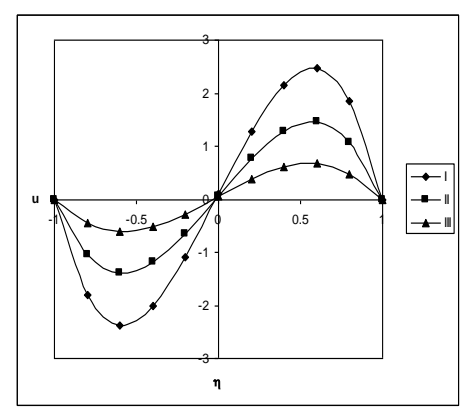

Fig. 1 : u with $\beta$ I II III $\begin{array}{llll}\beta & -0.3 & -0.7 & -0.9\end{array}$

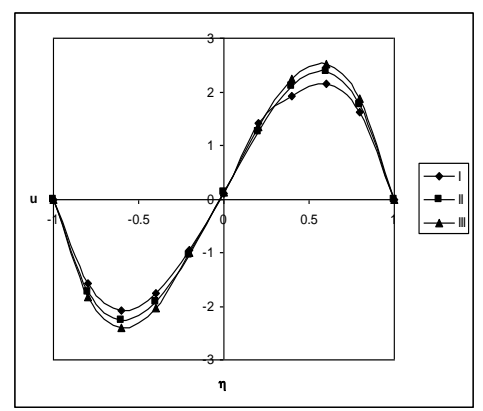

Fig. 3 : u with $\mathrm{N}$

I II III

N $2.5 \quad 5 \quad 10$

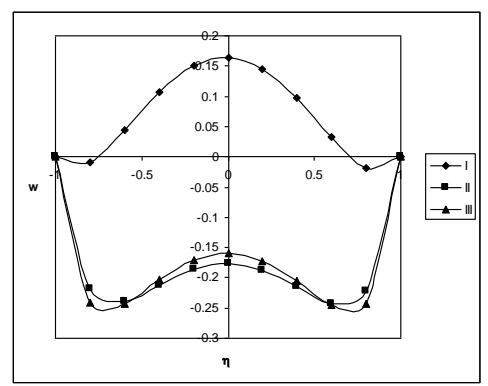

Fig. $5: w$ with $\lambda$

$\begin{array}{lll}\text { I II III } & \text { II }\end{array}$

$\begin{array}{llll}\lambda & 0.5 & 0.75 & 1.0\end{array}$

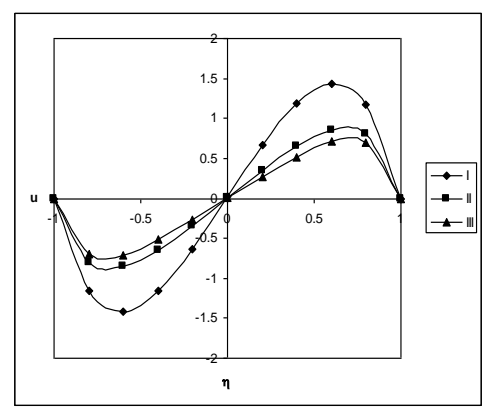

Fig. 2 : u with $\lambda$

$$
\begin{aligned}
& \text { I II III } \\
& \begin{array}{llll}
\lambda & 0.5 & 0.75 & 1.0
\end{array}
\end{aligned}
$$

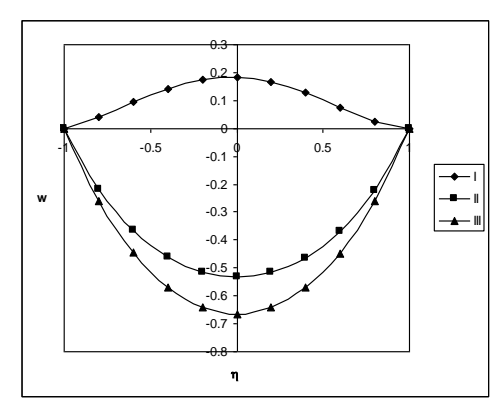

Fig. 4: w with $\beta$

$$
\text { I II III }
$$

$\begin{array}{lllll}\beta & -0.3 & -0.7 & -0.9\end{array}$

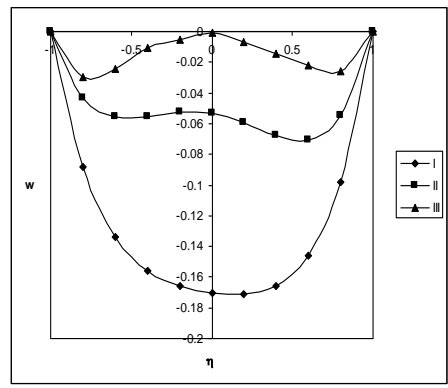

Fig. 6:w with $\mathrm{N}$

$$
\text { I II III }
$$

$\mathrm{N} 2.5 \quad 5 \quad 10$ 


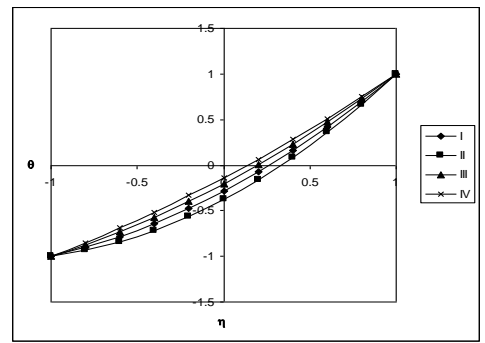

Fig. $7: \theta$ with $\beta$ $\begin{array}{lll}\text { I } & \text { II } & \text { III }\end{array}$ $\begin{array}{llll}\beta & -0.3 & -0.7 & -0.9\end{array}$

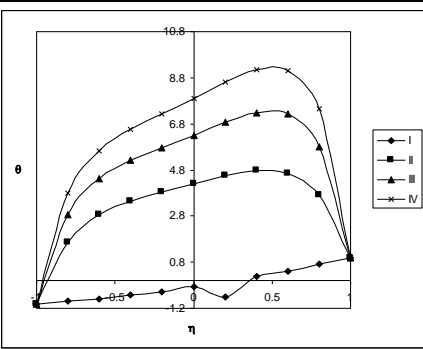

Fig. $8: \theta$ with $\lambda$

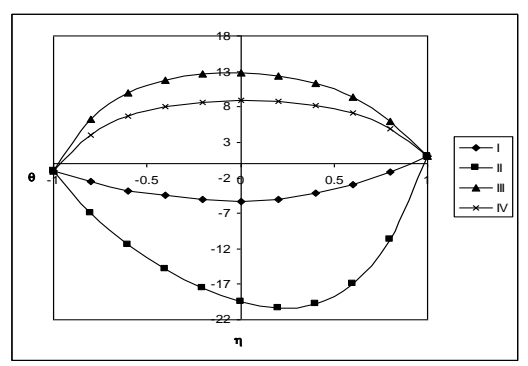

Fig.9 : $\theta$ with $\mathrm{N}$

I II III

$\mathrm{N}_{1} 2.55 \quad 10$

Table -1 Average Nusselt Number $(\mathrm{Nu})$ at $\eta=+1$

$\alpha=2, N=1, R=35, x=\pi / 4, t=\pi / 4, \gamma=2$

\begin{tabular}{|c|c|c|c|c|c|c|}
\hline $\mathbf{G}$ & I & II & III & IV & V & VI \\
\hline $\mathbf{1 0}^{\mathbf{3}}$ & 5.45938 & 10.27084 & 8.88499 & 42.29536 & 82.80968 & 150.70980 \\
\hline $\mathbf{3 x 1 0}^{\mathbf{3}}$ & 5.89852 & 16.37148 & 12.58187 & 30.68620 & 77.22723 & 174.43440 \\
\hline $\mathbf{- 1 0}^{\mathbf{3}}$ & 5.44009 & 11.41449 & 9.92020 & 20.61031 & 43.02969 & 73.49638 \\
\hline $\mathbf{- 3 x 1 0}^{\mathbf{3}}$ & 5.40651 & 10.58905 & 8.4256 & 19.51890 & 40.12225 & 67.28509 \\
\hline
\end{tabular}

Table -2 Average Nusselt Number $(\mathrm{Nu})$ at $\eta=-1$ $\alpha=2, N=1, R=35, x=\pi / 4, t=\pi / 4, \gamma=2$

\begin{tabular}{|c|c|c|c|c|c|c|}
\hline $\mathbf{G}$ & $\mathbf{I}$ & $\mathbf{I I}$ & $\mathbf{I I I}$ & $\mathbf{I V}$ & $\mathbf{V}$ & VI \\
\hline $\mathbf{1 0}^{\mathbf{3}}$ & -1.30169 & -1.41674 & -2.76975 & -11.19643 & -14.67373 & -20.76223 \\
\hline $\mathbf{3 \times 1 0}$ & -1.99740 & -3.11966 & 7.88410 & -5.89828 & -12.58028 & -24.72525 \\
\hline $\mathbf{- 1 0}^{\mathbf{3}}$ & -0.54529 & -1.78132 & 2.97253 & -2.30582 & -3.94302 & -5.73318 \\
\hline $\mathbf{- 3 x 1 0}^{\mathbf{3}}$ & -0.43892 & -1.55846 & -1.27973 & -1.91669 & -3.20869 & -4.56448 \\
\hline
\end{tabular}

\begin{tabular}{|l|l|l|l|l|l|l|}
\hline $\boldsymbol{\beta}$ & -0.3 & -0.7 & -0.9 & -0.5 & -0.5 & -0.5 \\
\hline $\boldsymbol{\lambda}$ & -0.5 & -0.5 & -0.5 & 0.5 & 0.75 & 1 \\
\hline
\end{tabular}




\section{References}

[1]. Christopher Philip, G, Heat and Mass transfer from a film into steady shear flow, J. I. Mech. Applied Matha, v. 43 (1990).

[2]. Lai F.C : Coupled heat and mass transfer by natural convection from a horizontal line source in saturated porous medium. Int. Comm. Heat Mass transfer, v. 17 pp, 489-499 (1990).

[3]. Chen, T.S, Yuh, C.F and Montsoglo, H : Combined Heat and Mass transfer in mixed convection along vertical and inclined planes. Int. J. Heat Mass transfer, v. 23, pp, (527-537) (1980).

[4]. Poulikakos, D. : On Buoyancy induced heat and mass transfer from a concentrated surface in a infinite porous medium, Int. J. Heat Mass transfer, v. 28, No. 3, pp, 621-629 (1985).

[5]. Angirasa, D, Peterson, G. P, Pop I : Combined heat and mass transfer by natural convection with buyancy effects in a fluid saturated porous medium, Int. J. Heat mass transfer v. 40, no. 12, pp, 2755-2773 (1997)

[6]. B. Hossain, M.D.A and H.S. Thakar: Radiation effects on mixed convection along a vertical plate with uniform surface temperature heat and mass transfer, V.34, PP. 243-248, (1996).

[7]. Ching-Yang Cheng : Natural convection Heat and Mass transfer near a vertical wavy surface with constant wall temperature and concentration in a porous medium, Int. Comm. Heat Mass transfer v. 27, No. 8, pp, 1143-1154 (2000).

[8]. Cess, R.D. The interaction of thermal radiation with free convection of heat transfer, Int. J. Heat Mass transfer v. 9, pp, 1269-1277 (1966). 